\title{
Periodontal Screening and Recording Code 2
}

National Cancer Institute

\section{Source}

National Cancer Institute. Periodontal Screening and Recording Code 2. NCI Thesaurus. Code C114650.

Indicates that all probing depth in the sextant is less than $3.5 \mathrm{~mm}$, but with supragingival or subgingival calculus and/or defective margins present. 